\title{
The lab on lockdown: thinking back and looking ahead
}

Gina R. Poe $e^{1}$ and Denise J. Cai ${ }^{2 凶}$

The shuttering of labs across nations during the COVID-19 pandemic created significant complexities related to remote lab management and ongoing research. The lessons learned may provide opportunities for new ways of working in the post-pandemic era.

'Lockdown' is a term that most of us likely never expected to be applied to our lives, much less to our labs. Originally used to characterize the confinement of prison inmates to their cells as an emergency safety measure and later the response to the threat of violence in other settings, the phrase describes an urgent and temporary situation. After what may have seemed an endless period of disruption, but was only a temporary closure, many of our labs are now in the process of re-opening. We were, of course, closed only in the physical sense. For many of us 'life in the lab' became 'lab-life at home'. As we prepare for what we hope will soon be a post-pandemic period, it is useful to reflect on the challenges we faced and lessons learned and to distill which of the 'temporary' changes that were made we can - and should - adopt more permanently.

While labs differ significantly, depending on research focus and resources, many of us experienced frustration and anxiety when it became clear that 'shut down' was necessary. As a junior faculty member, one of us had just set up equipment, secured funding and hired personnel to initiate the lab's first projects. As a senior faculty member, the other had long-term ongoing projects in progress and exciting new projects set to launch. Faculty at all levels certainly shared a sense of impatience over lost momentum. Nonetheless, as the threat posed by COVID-19 became evident ${ }^{1}$, concern for our teams' physical and mental health overcame anxiety about stalled research efforts. Ready or not, we shuttered the labs and began making plans for lab-life at home.

'Department of Integrative Biology and Physiology and Department of Psychiatry and Biobehavioral Sciences, Brain Research Institute, UCLA, Los Angeles, CA, USA.

${ }^{2}$ Nash Family Department of Neuroscience, Icahn School of Medicine at Mount Sinai, New York, NY, USA.

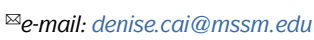
https://doi.org/10.1038/ s41583-020-0353-8

\section{What's that behind you?}

It became clear as we navigated this unprecedented landscape that flexibility would be key. In fact, there were many unexpected opportunities, especially in the ways we communicate with each other. In the early weeks of the shutdown, our labs formalized daily virtual meetings to talk about the business of working from home and to connect on a more personal level. These check-ins were important to keep up morale and to share the burden of worry and uncertainty.

Our virtual connections had us peering inside each other's personal spaces and lives in a new way and created a surprising intimacy. We found ourselves asking "what's that behind you?", waving to children who darted by, admiring cats perched on computer keyboards or listening to the strains of someone off-camera playing guitar.

Our work culture may be permanently, and positively, changed by this experience, especially with regard to parenting. It is easier to appreciate the necessity for children to figure into our work routines and plans when they have been, quite literally, in the picture. This understanding will be important as labs reopen while schools remain closed and childcare unavailable (or, for many trainees, unaffordable). At both of our institutions, leadership established a support fund to help defray the cost of financial hardship, including support for childcare. A colleague elsewhere created a Zoom story hour to give parents of young 'lab kids' a brief respite. Options for shared on-site childcare are being considered at multiple institutions. Thus, by blurring the lines between lab and living room, we may see innovative solutions for ongoing challenges that came more sharply into focus during the shutdown.

\section{Remote meetings and conferences}

One exciting aspect of working remotely has been the capacity to invite colleagues into our virtual labs to share research and form new collaborations. As our travel was arrested, we actually gained more freedom to engage with others globally. Through virtual lab meetings and journal clubs we connected better with colleagues around the world, reviewing a publication with a PI in Paris in the morning and convening with colleagues in Missoula, Montana in the afternoon. Our trainees had the opportunity to interact with PIs and peers that they may never have met in the pre-pandemic era. Opening our labs to each other in this way allowed us to expand and equalize our research 'reach' in ways we must not abandon in the post-COVID era.

We were also surprised to discover the excellence of virtual conferences and poster presentations, some of which were as engaging (if not more) than live events. One successful example was Neuromatch ${ }^{2}$, which offered 
both presentations and an international networking opportunity by 'mind matching' participants based on research interests. In the future, major professional society conferences could benefit from an expansion of virtual meeting formats and timespans, which, if done well, could enable people to attend who would otherwise be left out owing to cost or the infeasibility of travel, allow them to attend more as overlap between sessions is eliminated and reduce carbon emissions ${ }^{3,4}$. The costs and benefits of moving conferences to a virtual platform should be seriously considered by planners of all scientific meetings.

\section{Courses and open-source resources}

The transition to online classes also opened up new educational opportunities. Virtual platforms increase availability to students from all over the world, promoting access across labs, institutions and regions ${ }^{2}$. Lockdown also highlighted the need to develop methods for training and for teaching new techniques in the absence of hands-on, side-by-side activities (likely to continue even as labs reopen). We are creating video tutorials to supplement written protocols to facilitate skill and knowledge transfer and making them available to the community as open-source resources ${ }^{5}$. We hope that the trend of openly sharing techniques and tools will enhance the robustness of replication across labs and encourage innovation ${ }^{6}$. Creating greater access should become a priority in the post-pandemic era.

We note, however, that reliance on digital platforms reveals disparities and inequities in access for those who lack adequate computers, internet services and the personal space to take advantage of these opportunities. Delivering a professional presentation though a spotty cell phone connection in a noisy room, for example, is a challenge unequally shared. As virtual teaching and training becomes more widely adopted, we must also broaden access to the tools, spaces and services required to use them.

\section{Advancing the next generation}

Advancing trainees to their next career step constitutes an enormous, and rewarding, component of leading a lab. The shutdown has had substantial impact on this aspect of our work. Students and trainees on every level have had their plans disrupted, from high school interns and undergraduates lacking opportunities for in-lab experiences or the resources to engage in online learning, to graduate students who have deferred their education until in-person teaching can resume, to postdoctoral trainees whose career choices may be stalled by hiring freezes. Will those who plan a temporary leave from the lab return to the STEM path? We may also lose a generation of scientists as a result of other hardships the pandemic has created, including food and housing insecurity, loss of job opportunities and shifting family responsibilities. The long-term repercussions are difficult to predict, but as we assess the effectiveness of strategies for managing research and teams remotely, we also have an opportunity to think differently about inspiring young people to pursue STEM education and how to nurture careers in science as new crises evolve.

Finally, the intersection of the pandemic and the heightening of the Black Lives Matter movement inspired us to engage with our colleagues in hard but important conversations - reaffirming that Black lives do matter and discussing ways to fight racism, particularly in academia $^{7}$. We are participating in intra-institutional and inter-institutional discussions about racism in academia, utilizing online resources for self-education, supporting early career scientists and taking local action to support the Black Lives Matter movement. As we develop actionable plans in case of another shutdown, we are also developing strategies to improve the institutional climate of inclusiveness and to increase access and support for Black people and people of colour in our labs, institutions and the broader neuroscience community.

As researchers who study how the brain processes trauma, we know that responses to trauma in the past can predict future behaviour. There is no manual for 'managing your lab during a global pandemic' except the one we are creating through our daily experience. What we have learned from this challenging experiment will guide us to be more open to connecting with each other and to sharing ideas and resources as we move forward.

1. Mocco, J. Thoughts during a COVID nightmare. J. Neurointerv. Surg. 12, 637-638 (2020).

2. Achakulvisut, T. et al. Improving on legacy conferences by moving online. eLife 9, e57892 (2020).

3. Calisi, R. M., a Working Group of Mothers in Science. Opinion: how to tackle the childcare-conference conundrum. Proc. Natl Acad. Sci. USA 115, 2845-2849 (2018)

4. Swann, N. Are we there yet? eLife 8, e49202 (2019).

5. Pennington, Z. et al. ezTrack: an open-source video analysis pipeline for the investigation of animal behavior Sci. Rep. 9, 19979 (2019).

6. White, S. R., Amarante, L. M., Kravitz, A. V. \& Laubach, M. The future is open: open-source tools for behavioral neuroscience research. eNeuro 6, ENEURO.0223-19.2019 (2019).

7. Dzirasa, K. For Black scientists, the sorrow is also personal. Cell https://doi.org/10.1016/j.cell.2020.06.028 (2020).

\section{Acknowledgements}

We would like to thank S. Rabinowitz for editorial assistance. This work was supported by the NIH 1 R01MH60670 and R21 MH119020 from NIMH award to G.R.P. and the NIH Director's New Innovator Award 1DP2 MH122399, 1R01MH1 20162 from NIMH, One Mind Otsuka Rising Star Award, McKnight Memory and Cognitive Disorders Award, Klingenstein-Simons Fellowship Award in Neuroscience, Mount Sinai Distinguished Scholar Award, Brain Research Foundation Award and NARSAD Young Investigator Award to D.J.C.

Competing interests

The authors declare no competing interests. 